\title{
Impact of homocysteine levels on mortality risk in patients with chronic limb-threatening ischemia undergoing revascularization
}

\author{
Mitsuyoshi Takahara $^{1,2} \cdot$ Osamu lida $^{3} \cdot$ Yoshimitsu Soga $^{4} \cdot$ Akio Kodama $^{5} \cdot$ Hiroto Terashi $^{6} \cdot$ Nobuyoshi Azuma $^{7}$
}

Received: 25 March 2021 / Accepted: 14 May 2021 / Published online: 15 June 2021

(c) The Author(s) 2021

\begin{abstract}
The current study aimed to reveal the clinical impact of plasma homocysteine levels in chronic limb-threatening ischemia (CLTI) patients undergoing revascularization. This was a sub-analysis of a prospective multicenter registry of CLTI patients, named the Surgical reconstruction versus Peripheral INtervention in pAtients with critical limb isCHemia (SPINACH) study. The current analysis included 192 non-dialysis-dependent CLTI patients who underwent revascularization for CLTI, and whose plasma homocysteine levels at baseline were available. The association of clinical characteristics with homocysteine levels was evaluated with the linear regression model. The association of homocysteine levels with the mortality risk was investigated using the Cox proportional hazards regression model. Cystatin C-based estimated glomerular filtration rate (eGFR) was independently associated with log-transformed homocysteine levels; the adjusted standardized regression coefficient $(95 \%$ confidence interval) was $-0.432(-0.657$ to $-0.253 ; P<0.001)$. Homocysteine levels were significantly associated with the mortality risk in the univariate model $(P=0.017)$; the unadjusted hazard ratio was $1.71(1.13-2.50)$ per twofold increase. The association was significantly attenuated when adjusted for cystatin C-based eGFR $(P<0.001)$; the hazard ratio adjusted for cystatin C-based eGFR was $1.28(0.80-1.90 ; P=0.29)$. An apparent association of homocysteine levels with an increased risk of mortality could be explained by renal dysfunction. Future studies will be needed to validate the current findings.
\end{abstract}

Keywords Chronic limb-threatening ischemia $\cdot$ Homocysteine levels $\cdot$ Renal function $\cdot$ Cystatin C $\cdot$ Mortality risk

Osamu Iida

iida.osa@gmail.com

1 Department of Metabolic Medicine, Osaka University Graduate School of Medicine, 2-2 Yamadaoka, Suita 565-0871, Japan

2 Department of Diabetes Care Medicine, Osaka University Graduate School of Medicine, 2-2 Yamadaoka, Suita 565-0871, Japan

3 Cardiovascular Center, Kansai Rosai Hospital, 3-1-69 Inabaso, Amagasaki, Hyogo 660-8511, Japan

4 Department of Cardiology, Kokura Memorial Hospital, 3-2-1 Asano, Kokurakita-ku, Kitakyushu 802-0001, Japan

5 Division of Vascular Surgery, Department of Surgery, Nagoya University Graduate School of Medicine, 65 Tsurumai-cho, Showa-ku, Nagoya 466-8550, Japan

6 Department of Plastic Surgery, Kobe University Graduate School of Medicine, 7-5-2, Kusunoki-cho, Chuo-ku, Kobe 650-0017, Japan

7 Department of Vascular Surgery, Asahikawa Medical University, 2-1 Midorigaoka-higashi, Asahikawa 078-8510, Japan

\section{Introduction}

Chronic limb-threatening ischemia (CLTI) is the most advanced form of peripheral arterial disease, and patients with CLTI are at high risk of mortality, even after undergoing a timely revascularization [1]. Hyperhomocysteinemia is a well-known risk factor for atherosclerosis, especially for peripheral artery disease [2-4]. While the benefit of its correction by folate supplementation appear to be negligible [2-4], hyperhomocysteinemia per se has been regarded as a classic, useful biomarker for the risk of peripheral artery disease [2-4], and for clinical outcomes in a population with peripheral artery disease [5-7]. However, little was known about its impact on the mortality risk in CLTI patients undergoing revascularization.

The aim of the current study was to reveal the clinical impact of plasma homocysteine levels in CLTI patients undergoing revascularization. 


\section{Materials and methods}

We used a clinical database obtained from the Surgical reconstruction versus Peripheral INtervention in pAtients with critical limb isCHemia (SPINACH) study, a prospective, multicenter, observational study that registered patients with CLTI due to atherosclerotic arterial disease at 23 centers (12 vascular surgery departments and 11 interventional cardiology departments) in Japan $[1,8]$. CLTI patients were registered at the referral to the participating centers, between January 2012 and March 2013, and were scheduled to be followed-up for 3 years. The details of the SPINACH study are described elsewhere $[1,8]$. The study was performed in accordance with the Declaration of Helsinki and was approved by the ethics committee at the principal research institution, Asahikawa University Hospital (No. 1023) and all the other centers registering patients. Written informed consent was obtained from every participant. The current analysis included 192 nondialysis-dependent CLTI patients who underwent revascularization for either ischemic wound with the Wound, Ischemia, and foot Infection (WIfI) classification system [9] Ischemia (I) grade $2 / 3$ or ischemic rest pain with the WIfI I-3, and whose plasma homocysteine levels at baseline were available. Surgical reconstruction and endovascular therapy alone were selected in 69 and 122 patients, respectively. Skin perfusion pressures of $31-40 \mathrm{mmHg}$ and $\leq 30 \mathrm{mmHg}$ were treated as WIfI I-2 and 3, respectively [1]. The estimated glomerular filtration rate (eGFR) was calculated from the creatinine equation [10] and from the cystatin $\mathrm{C}$ equation [11].

\section{Statistical analysis}

Data are given as means and standard deviations or medians and interquartile ranges for continuous variables or as frequencies and percentages for discrete variables, respectively, if not otherwise mentioned. A $P$ value of less than 0.05 was considered statistically significant, and $95 \%$ confidence intervals are reported where appropriate. The association of clinical characteristics with homocysteine levels were evaluated with the linear regression model in which log-transformed homocysteine levels were treated as the dependent variable. The association with log-transformed homocysteine levels with the risk of all-cause mortality was investigated using the Cox proportional hazards regression model. We obtained the unadjusted hazard ratio from the univariate model, and the hazard ratio adjusted for each clinical characteristic from the bivariate model in which homocysteine levels and a covariate of interest were entered together. We additionally obtained the hazard ratio of log-transformed homocysteine levels for all-cause mortality with simultaneous adjustment for age, diabetes, smoking, and creatinine- and cystatin C-based eGFRs, using the multivariate Cox proportional hazards regression model. The association of homocysteine levels with the risk of major amputation and that of major adverse limb events (defined as a composite of major amputation and any reintervention) was analyzed using Fine and Gray's proportional hazards regression model for the sub-distribution of competing risks. Missing data were addressed using the multiple imputation by chained equations method. $\mathrm{P}$ values and $95 \%$ confidence intervals were obtained from 2000-time bootstrap resampling. All statistical analyses were performed using $\mathrm{R}$ version 3.6.0 (R Development Core Team, Vienna, Austria).

\section{Results}

Clinical characteristics of the study population are summarized in Table 1 . Mean age was $75 \pm 10$ years. Ischemic rest pain accounted for $15.2 \%$ and ischemic tissue loss accounted for the remaining $84.8 \%$. Median plasma homocysteine levels (interquartile range) were 12.8 (10.0-18.1) $\mathrm{nmol} / \mathrm{mL}$. Sixty-nine patients $(36.1 \%)$ underwent surgical reconstruction, and the remaining 122 (63.9\%) underwent endovascular therapy. As shown in Table 2, creatinine- and cystatin C-based eGFRs had a crude inverse association with log-transformed homocysteine levels; the unadjusted standardized regression coefficient (95\% confidence interval) was $-0.372(-0.513$ to -0.235$)$ and $-0.467(-0.592$ to -0.336 ), respectively (both $P<0.001$ ). In the multivariate regression model, cystatin C-based eGFR, but not creatinine-based eGFR or the other baseline characteristics, had an independent association with log-transformed homocysteine levels (Table 2).

During a median follow-up period of 2.8 (interquartile range 1.4-3.0) years, 62 patients died, and 65 patients experienced major adverse limb events, while 5 patients underwent major amputation. The univariate Cox proportional hazards regression model demonstrated that homocysteine levels were significantly associated with the mortality risk $(P=0.017)$; the unadjusted hazard ratio was 1.71 (1.13-2.50) per twofold increase. The hazard ratios adjusted for respective clinical characteristics are demonstrated in Fig. 1 . The hazard ratio was significantly attenuated when adjusted for cystatin C-based eGFR $(P<0.001)$; the hazard ratio adjusted for cystatin C-based eGFR was 1.28 $(0.80-1.90 ; P=0.29)$. On the other hand, the hazard ratio adjusted for creatinine-based eGFR was 1.54 (1.02-2.23; $P=0.042)$, which was not significantly different from the unadjusted hazard ratio $(P=0.097)$. The hazard ratio was not significantly changed from the unadjusted one when 
Table 1 Clinical characteristics of the study population

\begin{tabular}{|c|c|}
\hline $\mathrm{n}$ & 191 \\
\hline Age (years) & $75 \pm 10$ \\
\hline Male sex & $121(63.4 \%)$ \\
\hline \multicolumn{2}{|l|}{ Impaired mobility } \\
\hline None (self-ambulatory) & $116(60.7 \%)$ \\
\hline Requiring equipment & $66(34.6 \%)$ \\
\hline Requiring personal aid & $9(4.7 \%)$ \\
\hline Body mass index $\left(\mathrm{kg} / \mathrm{m}^{2}\right)$ & $21.9 \pm 3.4$ \\
\hline \multicolumn{2}{|l|}{ Smoking history } \\
\hline Never & $77(40.3 \%)$ \\
\hline Past & $78(40.8 \%)$ \\
\hline Current & $36(18.8 \%)$ \\
\hline Diabetes mellitus & $128(67.0 \%)$ \\
\hline Left ventricular ejection fraction (\%) & $64 \pm 11$ \\
\hline (Missing data) & $7(3.7 \%)$ \\
\hline Creatinine-based eGFR $\left(\mathrm{mL} / \mathrm{min} / 1.73 \mathrm{~m}^{2}\right)$ & $60 \pm 28$ \\
\hline Cystatin C-based eGFR $\left(\mathrm{mL} / \mathrm{min} / 1.73 \mathrm{~m}^{2}\right)$ & $55 \pm 24$ \\
\hline (Missing data) & $13(6.8 \%)$ \\
\hline Hemoglobin levels (g/dL) & $11.6 \pm 2.1$ \\
\hline Albumin levels (g/dL) & $3.5 \pm 0.6$ \\
\hline (Missing data) & $1(0.5 \%)$ \\
\hline Cholinesterase (U/L) & $239 \pm 75$ \\
\hline (Missing data) & $4(2.1 \%)$ \\
\hline Homocysteine levels (nmol/mL) & $12.8(10.0-18.1)$ \\
\hline \multicolumn{2}{|l|}{ Medication use } \\
\hline Statin use & $59(30.9 \%)$ \\
\hline Renin-angiotensin system inhibitor use & $102(53.4 \%)$ \\
\hline Beta blocker use & $36(18.8 \%)$ \\
\hline Aspirin use & $118(61.8 \%)$ \\
\hline Thienopyridine use & $57(29.8 \%)$ \\
\hline Cilostazol use & $63(33.0 \%)$ \\
\hline Insulin use & $40(20.9 \%)$ \\
\hline Oral antidiabetic medication use & $59(30.9 \%)$ \\
\hline History of intermittent claudication & $116(61.1 \%)$ \\
\hline (Missing data) & $1(0.5 \%)$ \\
\hline \multicolumn{2}{|l|}{ WIfI Wound grade } \\
\hline $\mathrm{W}-0$ & $29(15.2 \%)$ \\
\hline $\mathrm{W}-1$ & $53(27.7 \%)$ \\
\hline $\mathrm{W}-2$ & $80(41.9 \%)$ \\
\hline $\mathrm{W}-3$ & $29(15.2 \%)$ \\
\hline \multicolumn{2}{|l|}{ WIfI Ischemia grade } \\
\hline $\mathrm{I}-2$ & $28(14.7 \%)$ \\
\hline $\mathrm{I}-3$ & $163(85.3 \%)$ \\
\hline \multicolumn{2}{|l|}{ WIfI Foot infection grade } \\
\hline fI-0 & $106(55.5 \%)$ \\
\hline fI-1 & $48(25.1 \%)$ \\
\hline $\mathrm{fI}-2$ & $36(18.8 \%)$ \\
\hline fI-3 & $1(0.5 \%)$ \\
\hline Bilateral CLTI & $28(14.7 \%)$ \\
\hline \multicolumn{2}{|l|}{ Revascularization strategy } \\
\hline Surgical reconstruction & $69(36.1 \%)$ \\
\hline Endovascular therapy & $122(63.9 \%)$ \\
\hline
\end{tabular}

Data are mean \pm standard deviation, median (interquartile range) or frequency (percentage) adjusted for any of the other clinical characteristics (Fig. 1). The hazard ratio simultaneously adjusted for age, smoking, diabetes, and creatinine- and cystatin C-based eGFRs was $1.26(0.74-2.02 ; P=0.41)$. Homocysteine levels were not significantly associated with the risk of major amputation or that of major adverse limb events; the unadjusted hazard ratios per twofold increase were $1.50(0.26-5.21 ; P=0.56)$ and $0.97(0.68-1.38 ; P=0.86)$, respectively.

\section{Discussion}

The current study demonstrated the clinical impact of plasma homocysteine levels in non-dialysis-dependent CLTI patients undergoing revascularization. Homocysteine levels were significantly associated with cystatin C-based eGFR. Homocysteine levels were significantly associated with an increased risk of mortality, but the association was attenuated when adjusted for cystatin C-based eGFR.

Homocysteine is an endogenous sulfur-containing amino acid intermediate of the essential amino acid methionine, and is not obtained directly from the diet [12]. An excessive amount of homocysteine will increase the oxidative stress and induce endothelial dysfunction and vessel injury, potentially accelerating atherosclerosis [13]. Its elevated plasma levels have been, therefore, expected to reflect systemic proatherosclerotic status and to be a useful biomarker related to cardiovascular disease [12]. On the other hand, in vivo, circulating homocysteine is filtered and metabolized by the kidneys in large amounts; decreased renal function will elevate plasma homocysteine levels [12]. The current finding of an inverse correlation between plasma homocysteine levels and eGFR was in line with this story.

Plasma homocysteine levels were associated with an increased mortality risk, but the association was attenuated after adjustment for cystatin C-based eGFR. This attenuation suggests that the prognostic impact of elevated plasma homocysteine levels would be explained by decreased renal function. Renal dysfunction is a well-known predictor of poor prognosis in a CLTI population [14], and hyperhomocysteinemia might be just a marker of renal dysfunction. In the prediction of the mortality risk, homocysteine levels might not provide more information than was provided by renal function. Future studies with a larger sample size will be needed to validate the current findings.

On the other hand, elevated plasma homocysteine levels were still significantly associated with the mortality risk when instead adjusted for creatinine-based eGFR. Creatinine-based eGFR would be inferior to cystatin C-based eGFR in accurate estimation of renal function [15]. Plasma homocysteine levels, as a marker of renal dysfunction, would compensate creatinine-based eGFR for its inaccuracy, and keep their significant association with the mortality risk in 
Table 2 Association of clinical characteristics with homocysteine levels

\begin{tabular}{lll}
\hline & Unadjusted standardized regression coefficients & Adjusted standardized regression coefficients \\
\hline Age & $0.037[-0.131$ to 0.203$](P=0.67)$ & $-0.099[-0.270$ to 0.072$](P=0.28)$ \\
Male sex & $-0.033[-0.183$ to 0.110$](P=0.66)$ & $0.026[-0.154$ to 0.178$](P=0.78)$ \\
Body mass index & $-0.013[-0.160$ to 0.137$](P=0.89)$ & $0.008[-0.118$ to 0.148$](P=0.87)$ \\
Impaired mobility & $0.066[-0.112$ to 0.239$](P=0.48)$ & $0.071[-0.098$ to 0.235$](P=0.46)$ \\
Smoking & $0.025[-0.115$ to 0.167$](P=0.71)$ & $0.086[-0.068$ to 0.261$](P=0.26)$ \\
Diabetes mellitus & $0.021[-0.126$ to 0.166$](P=0.77)$ & $-0.015[-0.155$ to 0.123$](P=0.83)$ \\
Left ventricular ejection fraction & $-0.034[-0.146$ to 0.086$](P=0.59)$ & $0.069[-0.048$ to 0.190$](P=0.26)$ \\
Creatinine-based eGFR & $-0.372[-0.513$ to -0.235$](P<0.001)$ & $-0.092[-0.298$ to 0.152$](P=0.46)$ \\
Cystatin C-based eGFR & $-0.467[-0.592$ to -0.336$](P<0.001)$ & $-0.432[-0.657$ to -0.253$](P<0.001)$ \\
Hemoglobin levels & $-0.094[-0.211$ to 0.021$](P=0.11)$ & $0.009[-0.123$ to 0.155$](P=0.84)$ \\
Albumin levels & $-0.072[-0.193$ to 0.056$](P=0.28)$ & $0.036[-0.133$ to 0.212$](P=0.65)$ \\
Cholinesterase levels & $-0.073[-0.219$ to 0.072$](P=0.34)$ & $-0.032[-0.213$ to 0.143$](P=0.70)$ \\
History of claudication & $-0.138[-0.281$ to 0.007$](P=0.061)$ & $-0.077[-0.208$ to 0.067$](P=0.28)$ \\
WIfI-W grade & $0.060[-0.099$ to 0.223$](P=0.47)$ & $0.065[-0.126$ to 0.246$](P=0.53)$ \\
WIfI-I grade & $-0.095[-0.250$ to 0.059$](P=0.22)$ & $-0.066[-0.205$ to 0.087$](P=0.43)$ \\
WIfI-fI grade & $-0.103[-0.247$ to 0.045$](P=0.17)$ & $-0.136[-0.279$ to 0.021$](P=0.094)$ \\
Bilateral CLTI & $0.129[-0.056$ to 0.310$](P=0.16)$ & $0.122[-0.037$ to 0.274$](P=0.13)$ \\
Endovascular therapy vs surgical recon- & $0.102[-0.026$ to 0.231$](P=0.10)$ & $0.061[-0.076$ to 0.189$](P=0.37)$ \\
$\quad$ struction & &
\end{tabular}

Data are presented as standardized regression coefficients for log-transformed homocysteine levels [95\% confidence intervals] $(P$ values). Adjusted standardized regression coefficients were obtained from the multivariate linear model in which all the variables listed in the table were entered as the explanatory variables

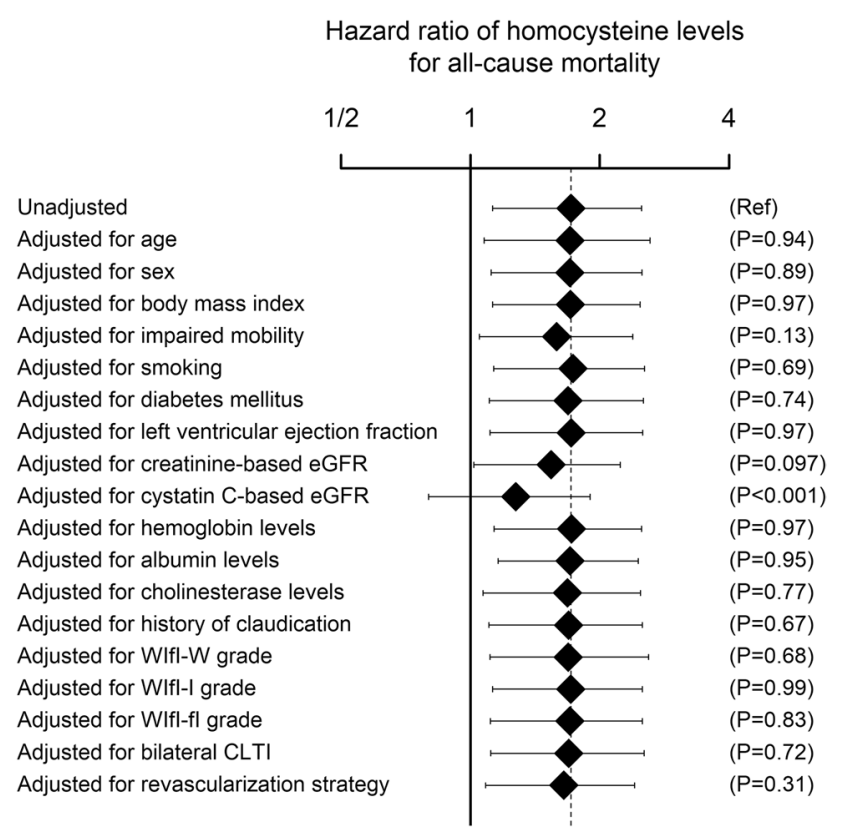

Fig. 1 Hazard ratio of homocysteine levels for all-cause mortality. Data are hazard ratios of homocysteine levels (per twofold increase) for all-cause mortality, with adjustment for each covariate. Error bars indicate $95 \%$ confidence intervals. The dotted line as well as the top diamond shows the unadjusted hazard ratio of homocysteine levels (per twofold increase) for all-cause mortality. $P$ values are for the difference between the hazard ratio adjusted for respective covariates and the unadjusted hazard ratio the bivariate model including creatinine-based eGFR as a covariate.

The current study had some limitations. First, data were not available on clinical variables potentially influencing plasma homocysteine levels, such as folate and B vitamin deficiencies, their supplementation, and the dietary consumption of methionine-containing protein. Second, the glomerular filtration rate was not directly measured, but was estimated with the use of creatinine and cystatin C levels. Third, the current study analyzed Japanese CLTI patients. Future studies on other ethnics will be needed to validate the current findings.

In conclusion, the current study investigated the clinical impact of plasma homocysteine levels in non-dialysisdependent CLTI patients undergoing revascularization. The association of homocysteine levels with the mortality risk lost statistical significance after adjustment for cystatin C-based eGFR. An apparent association of homocysteine levels with an increased risk of mortality could be explained by renal dysfunction. Future studies will be needed to validate the current findings.

Acknowledgements The SPINACH Study is sponsored by Abbott Vascular Japan Co., Ltd, Boston Scientific Japan K.K., Cook Japan Incorporated, Goodman Co., Ltd, Johnson \& Johnson K.K., Kaken Pharmaceutical Co., Ltd, Kaneka Medix Corporation, Medicon Inc., Medikit Co., Ltd, Medtronic Japan Co., Ltd, Mitsubishi Tanabe Pharma Corporation, MSD K.K., St. Jude Medical Japan Co., Ltd, Taisho Toyama 
Pharmaceutical Co., Ltd, Terumo Corp., W.L. Gore \& Associates, Co., Ltd (in alphabetical order). The funding companies played no role in the design of the study, selection of the enrolled patients, revascularization procedures or equipment, or interpretation of the data.

\section{Declarations}

Conflict of interest The authors declare that they have no conflict of interest.

Open Access This article is licensed under a Creative Commons Attribution 4.0 International License, which permits use, sharing, adaptation, distribution and reproduction in any medium or format, as long as you give appropriate credit to the original author(s) and the source, provide a link to the Creative Commons licence, and indicate if changes were made. The images or other third party material in this article are included in the article's Creative Commons licence, unless indicated otherwise in a credit line to the material. If material is not included in the article's Creative Commons licence and your intended use is not permitted by statutory regulation or exceeds the permitted use, you will need to obtain permission directly from the copyright holder. To view a copy of this licence, visit http://creativecommons.org/licenses/by/4.0/.

\section{References}

1. Iida O, Takahara M, Soga Y, Kodama A, Terashi H, Azuma N, Investigators S (2017) Three-year outcomes of surgical versus endovascular revascularization for critical limb ischemia: the SPINACH Study (surgical reconstruction versus peripheral intervention in patients with critical limb ischemia). Circ Cardiovasc Interv 10:e005531

2. Dormandy JA, Rutherford RB (2000) Management of peripheral arterial disease (PAD). TASC Working Group. TransAtlantic Inter-Society Consensus (TASC). J Vasc Surg 31:S1-S296

3. Norgren L, Hiatt WR, Dormandy JA, Nehler MR, Harris KA, Fowkes FG, Group TIW (2007) Inter-society consensus for the management of peripheral arterial disease (TASC II). J Vasc Surg 45(Suppl S):S5-67

4. Conte MS, Bradbury AW, Kolh P, White JV, Dick F, Fitridge R, Mills JL, Ricco JB, Suresh KR, Murad MH, Group GVGW (2019) Global vascular guidelines on the management of chronic limbthreatening ischemia. J Vasc Surg 69:3S-125S.e140

5. Kumakura H, Fujita K, Kanai H, Araki Y, Hojo Y, Kasama S, Iwasaki T, Ichikawa S, Nakashima K, Minami K (2015) High-sensitivity C-reactive Protein, Lipoprotein(a) and homocysteine are risk factors for coronary artery disease in Japanese Patients with Peripheral Arterial Disease. J Atheroscler Thromb 22:344-354

6. McDermott MM, Liu K, Ferrucci L, Tian L, Guralnik JM, Green D, Tan J, Liao Y, Pearce WH, Schneider JR, McCue K, Ridker P,
Rifai N, Criqui MH (2008) Circulating blood markers and functional impairment in peripheral arterial disease. J Am Geriatr Soc 56:1504-1510

7. Laxdal E, Wirsching J, Pedersen G, Bertz A, Amundsen SR, Dregelid E, Jonung T, Nyheim T, Aune S (2006) Homocysteine levels, haemostatic risk factors and patency rates after endovascular treatment of the common iliac arteries. Eur J Vasc Endovasc Surg 31:244-250

8. Azuma N, Iida O, Takahara M, Soga Y, Kodama A (2014) Surgical reconstruction versus peripheral intervention in patients with critical limb ischemia-a prospective multicenter registry in Japan: The SPINACH study design and rationale. Vascular 22:411-420

9. Mills JL Sr, Conte MS, Armstrong DG, Pomposelli FB, Schanzer A, Sidawy AN, Andros G (2014) The Society for Vascular Surgery Lower Extremity Threatened Limb Classification System: risk stratification based on wound, ischemia, and foot infection (WIfI). J Vasc Surg 59(220-234):e1-2

10. Matsuo S, Imai E, Horio M, Yasuda Y, Tomita K, Nitta K, Yamagata K, Tomino Y, Yokoyama H, Hishida A, Collaborators developing the Japanese equation for estimated GFR (2009) Revised equations for estimated GFR from serum creatinine in Japan. Am J Kidney Dis 53:982-992

11. Horio M, Imai E, Yasuda Y, Watanabe T, Matsuo S, Collaborators Developing the Japanese Equation for Estimated GFR (2013) GFR estimation using standardized serum cystatin C in Japan. Am J Kidney Dis 61:197-203

12. Friedman AN, Bostom AG, Selhub J, Levey AS, Rosenberg IH (2001) The kidney and homocysteine metabolism. J Am Soc Nephrol 12:2181-2189

13. Tanriverdi H, Evrengul H, Enli Y, Kuru O, Seleci D, Tanriverdi S, Tuzun N, Kaftan HA, Karabulut N (2007) Effect of homocysteineinduced oxidative stress on endothelial function in coronary slowflow. Cardiology 107:313-320

14. Azuma N, Takahara M, Kodama A, Soga Y, Terashi H, Tazaki J, Yamaoka T, Koya A, Iida O (2019) Predictive model for mortality risk including the wound, ischemia, foot infection classification in patients undergoing revascularization for critical limb ischemia. Circ Cardiovasc Interv 12:e08015

15. Shlipak MG, Matsushita K, Arnlov J, Inker LA, Katz R, Polkinghorne KR, Rothenbacher D, Sarnak MJ, Astor BC, Coresh J, Levey AS, Gansevoort RT, Consortium CKDP (2013) Cystatin C versus creatinine in determining risk based on kidney function. $\mathrm{N}$ Engl J Med 369:932-943

Publisher's Note Springer Nature remains neutral with regard to jurisdictional claims in published maps and institutional affiliations. 